\title{
Experiencing Flow: An Interpersonal Process View
}

\author{
${ }^{1}$ Woosong University, Daejeon, Rep. of Korea \\ *Min Han, E-mail: rainmaster44@gmail.com
}

Min $\operatorname{Han}^{1 *}$

\begin{abstract}
Previous researches on Flow have focused on the intrapersonal aspect through which individuals can experience Flow. The balance between challenge and skill has been the most important precondition for Flow. However, Flow may be attainable through other methods especially in terms of historical and cultural perspectives. To provide an alternative explanation, the current study discussed Shinmyeong, a cultural phenomenon in Korea, and its specific process as a cultural form of Flow. People who experienced Shinmyeong reported that they began to experience it when they interacted with the others who were in the same place at the time of Shinmyeong experience. They thought every person there was exchanging the same feelings with one another and the feelings were amplified based on such beliefs. This interpersonal process regulated the intensity of Shinmyeong, and the way people experience it was constructed by culture. The cultural concept of Woori (we) was also discussed to understand the way of communication among Korean people, which may be the foundation of the interpersonal process in
\end{abstract} Shinmyeong experience.

\section{Keywords}

flow, Shinmyeong, precondition, interpersonal process, culture

\section{Introduction}

In psychology, flow is not a recent phenomenon. Flow was identified to be commonly present across widely diverse activities. Flow is known as the mental state of operation in which a person engaging in an activity is totally immersed in the feelings of energized focus, full involvement, and enjoyment in the process of the activity. In essence, flow is characterized as a complete absorption in what one does. According to Csikszentmihalyi, flow is a completely focused motivation. It is a single-minded immersion and represents perhaps the ultimate experience in harnessing the emotions in the service of performing and learning. In flow, the emotions are not just contained and channeled, but positive, energized, and aligned with the task at hand. The hallmark of flow is a feeling of spontaneous joy, even rapture, while performing a task. On one hand, flow is also described as a deep focus on nothing but the activity—not even oneself or one's emotions (Csikszentmihalyi, 1975; Csikszentmihalyi, 1988).

Experiencing flow is based on autotelic motivations. In other words, people engage in certain activities because those activities alone are rewarding. While experiencing flow, people are so absorbed in the activities that they become unconscious of themselves. During this experience, they feel positive 
emotions (Csikszentmihalyi, 1988). In positive psychology, which was suggested to help people find their way to happiness (Seligman \& Csikszentmihalyi, 2000), flow is an important subject because people who experience flow describe to be in the states of ecstasy, rapture, and so on. According to Csikszentmihalyi (1988), people who had a taste of this extreme happiness tend to engage in the same activities again to relive the feeling. Even though he explained this autotelic process in the context of flow with a limited set of activities, the process itself can be observed in all events that allow people to have such experiences. Furthermore, Csikszentmihalyi (2004) himself acknowledged the existence of other possible ways to reach great happiness. Considering history and various cultures, gambling, drugs, sex, and religion are only a few examples in which people achieve intense joy and pleasure. Experiencing flow is one specific way of doing so.

Establishing the concept of flow, Csikszentmihalyi (1988) focused on individual activities such as playing chess, climbing mountains, artistic performance, medical operation, and so on. To experience flow while engaging in these activities, some preconditions have to be satisfied. These are clear goals, immediate feedback, and balance of skill and challenge. Clear goals and immediate feedback help people concentrate on their activities. When the individuals' skills are compatible with the degree of difficulty, experiencing flow becomes possible. Following Csikszentmihalyi's explanations, the last precondition may be most crucial in initiating flow. People will not get into flow if their abilities and the challenge of the activity are not in harmony even if the other two preconditions are satisfied. This idea has been supported by other researchers repeatedly (Massimini \& Carli, 1988; LeFevre, 1988).

\section{A Question for the Precondition of Flow}

For the last decades of flow research, the basic assumptions and preconditions of flow have been accepted without doubts since Csikszentmihalyi (1988) introduced them. However, Csikszentmihalyi $(1997,2004)$ himself recently raised questions concerning these and he tried to expand the concept of flow from individual activities with autotelic motivations to general contexts of everyday life. This leads to the question: How can flow be experienced in ordinary circumstances? All explanations so far for experiencing flow have focused on the balance of challenge and skill, which takes place at the intrapersonal level. However, in everyday life, individuals participate in various activities in social context that may include interactions with others. This interpersonal process may be crucial in examining flow experience in daily life situations.

As Csikszentmihalyi $(1991,1998)$ wrote, people engage in countless activities that do not require specific skills mostly because they do not consider these activities as challenges. For example, Korean elderly people reported experiencing flow in social and recreational activities (Han, 1988). In Sato's research (1988), Japanese motorcycle gangs described having a feeling similar to flow without mentioning the terms such as balance, skill, or difficulty. They rather characterized their experience as having found an outlet. In other words, the flow experience of the gangs seemed to have its origin in the desire to overcome frustration and to show self-related values rather than in the activity itself. 
These types of flow are somewhat different from the traditional definition of flow. In Han's work (1988), flow was assessed using the terms that Korean elderly people were accustomed to (e.g., concentration or involving). Yet those were important characteristics of the Shinmyeong experience that will be discussed later in this article (Han \& Han, 2007b). In the case of Sato's study (1988), the experiences of motorcycle gangs were classified as flow because the terms such as "speed" and "thrill" could be related to "skill" and "challenge" (Tamura \& Mugishima, 1975). Regardless of cultural context, all human activities that can provide pleasure might be considered as challenge and people should have adequate skills to find happiness in the activities involved. While chatting with friends, for instance, the individuals need social skills to lead conversations in order to be joyful. Finding pleasure in an activity seems to be a personal task. Or is there an alternative?

\section{Another Way to Experience Flow}

There might be a number ways to get into the state of flow. Historically, mankind has developed various ways to become happy regardless of cultures. Some of them are individual, some of them are collective. From this perspective, Csikszentmihalyi's explanation for flow just describes one possible way, especially on individual level. In most cases, the theory of flow is insufficient to fully explain the cause of pleasure because it limits the events through which people can get into flow to individual activities. The theory is only useful for explaining the intrapersonal perspective while experiencing happiness.

However, if we try to expand the concept of flow to general contexts as Csikszentmihalyi $(1991,1998$, 2004) did, we need to pay attention to the related conditions or processes. Researchers can learn how the phenomena of flow are interpersonally embedded by studying non-Western societies because Eastern societies have developed the ways to make their people and the whole society become happy. For this purpose, the Korean concept of Shinmyeong is introduced here. It is the name of cultural emotion which refers to a great pleasure. By understanding the structure and processes of Shinmyeong, it is possible to provide a new basis for expanding the concept of flow in psychological theorization.

\section{Flow and Shinmyeong}

Shinmyeong is known as the most ideal psychological experience in Korean culture (Han \& Han, 2007a). People who experienced Shinmyeong described their experiences as the greatest happiness - using various expressions. Examples for the expressions used are "forgetting oneself" (Oh, 1994), "merging of action and awareness" (Yoo \& Kim, 1997), "ecstasy" (Kim, Kim, Hong, Choi, Kim, Yoo, Lee, Seo, Lee, \& Seo, 1982), or "time distortion" (Oh, 1994). All these terms are similar to the ones found in relation to the state of flow. The following excerpt could also have been taken from a flow study: When the passion of playing became overwhelming, the ego vanished. In this moment, I was no longer aware of my own playing, basic emotions like pleasure, anger, sadness, ...became meaningless. The state can be described as pure nothing (Duksoo Kim, 1994). 
Its origin is, nevertheless, an interview of a famous Korean traditional musician who described his Shinmyeong experience during his performance. Despite the conceptual difference where flow was conceptualized by researchers and Shinmyeong is a cultural phenomenon (Han \& Han, 2007a), flow and Shinmyeong share some aspects in common. Possibly, these similarities between flow and Shinmyeong support Csikszentmihalyi's opinion (1991) that the way pleasure is experienced is a general one irrespective of its sources. Therefore, if we focused on Shinmyeong, we could find alternative explanations for expanding the notion of flow.

\section{How Do People Experience Shinmyeong?}

Because of its cultural and historical background, Shinmyeong is experienced in remarkably diverse contexts. The contexts that influence people to experience Shinyeong can be categorized into three types (see Table 1), although Korean people have experienced it in various contexts for a profoundly long time.

Table 1. Types of Shinmyeong Experiences (Han \& Han, 2007b)

\begin{tabular}{|c|c|c|}
\hline types & frequency $(\%)$ & examples of cases \\
\hline $\begin{array}{l}\text { by } \quad \text { self-related } \\
\text { events }\end{array}$ & $71(32.7)$ & $\begin{array}{l}\text { victory of Korean national teams, come-from-behind victory, } \\
\text { winning a lottery, passing exams, getting promotions, giving a } \\
\text { birth to own baby, winning prizes, getting a job, etc. }\end{array}$ \\
\hline by co-experience & $73(33.1)$ & $\begin{array}{l}\text { cheering for own team, college festivals, traditional festivals, } \\
\text { playing traditional music, cooperating with own group, parties } \\
\text { with friends, trips with family(or friends), etc. }\end{array}$ \\
\hline by self-expression & $43(19.5)$ & $\begin{array}{l}\text { exhibitions, artistic performances, climbing mountain, finishing } \\
\text { marathon full course, leading own projects, etc. }\end{array}$ \\
\hline
\end{tabular}

The table shows three types of Shinmyeong which were classified by the kinds of events people experienced. First, people experience Shinmyeong from the events by which their own values are maximized. Second, in the specific situations in which people feel oneness with others, they experience Shinmyeong. Lastly, when people feel personal meaning during some kinds of performances, they reported having Shinmyeong.

As can be seen, Koreans experience Shinmyeong for diverse reasons. However, according to the results of the same study (Han \& Han, 2007b), the way people experienced Shinmyeong was a common one, independent of the types. Based on the Grounded theory, a theoretical model was derived out of the data collected from the people whom experienced Shinmyeong. Figure 1 shows the result of axial coding (Strauss \& Corbin, 1998). The processes of Shinmyeong experience can be summarized as follows. 
The moment people are starting to experience Shinmyeong is when their self-worth is maximized (causal condition). The momentary feeling is affected by their past self-worth (contextual condition). In other words: The lower their self-worth was, the greater is the difference to its maximized version and the stronger is the related feeling (phenomenon). The experience of Shinmyeong can also be influenced by the existence of other people whom are involved in same context (intervening condition). Within groups, people who are experiencing Shinmyeong can express their feelings in various ways (action/interaction). The expressive phase can be amplified by people's interactions and interpersonal processes. Finally, as a result of all these processes, people can experience many positive effects such as a sense of freedom, positive motivation for the future, etc. (consequence) (Han \& Han, 2007b).

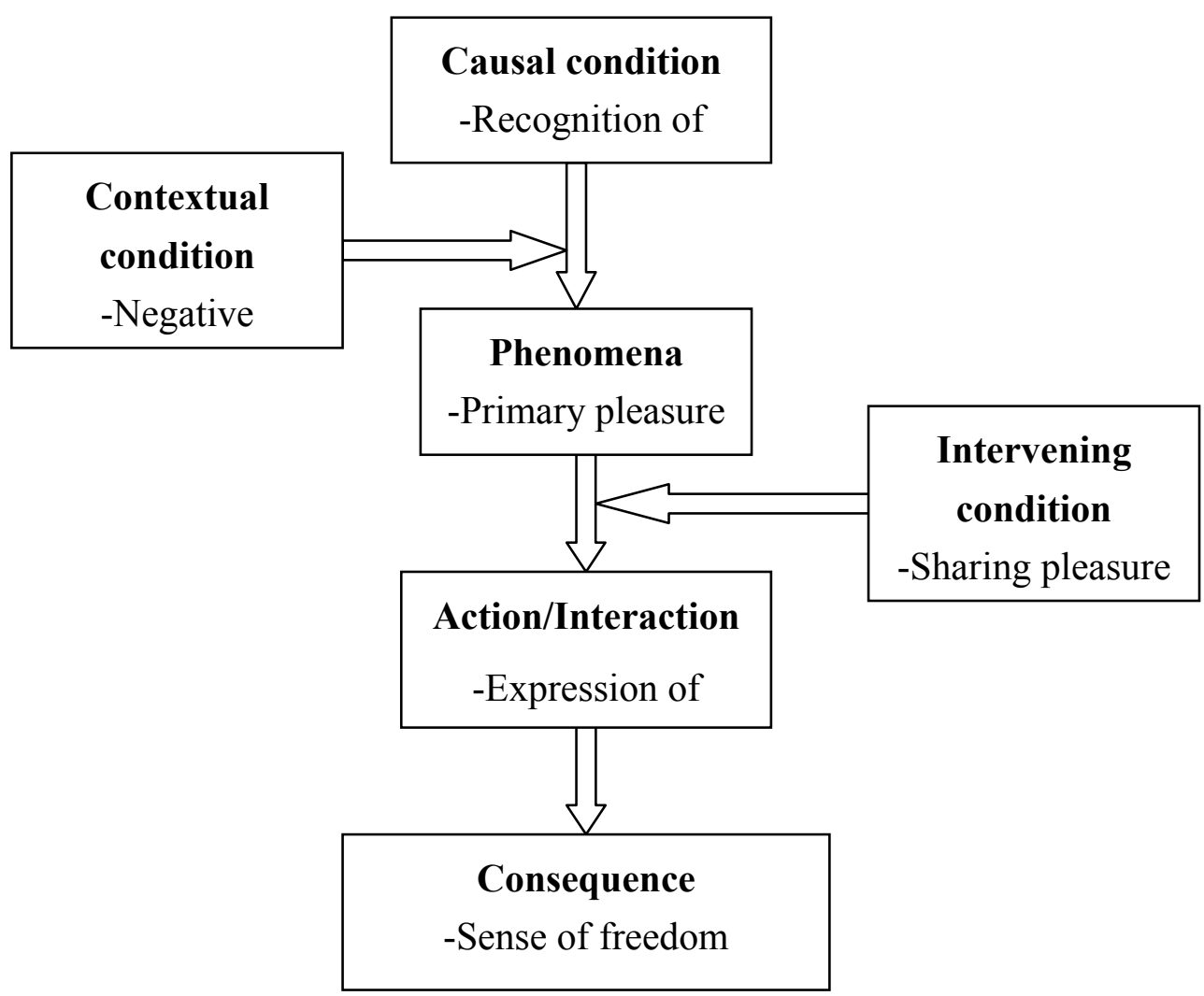

Figure 1. A Functional Account of Shinmyeong Experience

Among these processes, the phase "action/interaction" is most similar to the state of flow. In this phase, the feeling that originates in the recognition of self-related values and is influenced by interactions with others can be maximized by all kinds of expressive actions. Continuing interactions during the expression phase can even amplify the feeling. That is the peak state of Shinmyeong. 


\section{Preconditions for the Shinmyeong Experience}

Shinmyeong mainly differs from flow in its preconditions. According to the model introduced above, people start to experience Shinmyeong when their self-related values are recognized, but the following processes (co-experience and expressive phase) are vital for reaching the peak state of Shinmyeong. For flow, the match of skill and challenge is the vital condition. In both cases, the feeling of the peak state is so strong and rewarding that people want to experience it again. It is the logic of Csikszentmihalyi's autotelic motive (1988) that can be applied to all kinds of positive experiences, not just to challenging activities.

To find another path to great happiness, "Shinmyeong by self-expression", a particular type of Shinmyeong, is more closely examined. Because it is based on the same activities as flow (e.g., artistic performances), the comparison of the preconditions for the peak state is easier. Of course, the fact that Shinmyeong by self-expression and flow share some similar contexts in which people experienced them does not mean that we are looking at the same experiences.

In Sinmyeong, people describe their experiences with terms related to self-worth, whereas the terms used for the description of flow are limited to individual states. Examples for the description of Sinmyeong are the followings (Han, 2007b).

"Then I became aware of the audience and their reactions, I mean, they seemed to feel what I wanted to express. I felt empathic with them. That made me more excited." $<$ ID 26>

"When we played jazz, when I got to try something I had not tried before, when my colleagues performed well. Also, when the audience seemed to enjoy my efforts, these moments led me to another world. " $<$ ID 69>

This data was classified as "intervening" condition with the main characteristic as the active interaction between the person who is experiencing Shinmyeong and the other people who are in the same place at the same time. While performing, they were paying attention not only to the performance itself but also to the responses of colleagues and audiences. Other examples of this phase are as follows.

There was a certain moment when all players almost closed their eyes. It might have been caused by a tune we were playing. Anyway, at the moment we saw each other, we felt what the other one felt, ...(then we got into Shinmyeong.) $<I D 2>$

When I noticed that the player who was sitting next to me was performing passionately, it affected my performance. In other words, I started to try playing much better. Then we felt that our performances were in perfect harmony, our feelings were amplified. $<I D$ 37>

Time distortion is one of the notable features of flow (Csikszentmihalyi, 1988). While experiencing flow, people are so immersed in what they are doing that they do not perceive time as usual. The exact same phenomenon can be observed in the Shinmyeong experience. However, the explanation for this phenomenon is somewhat different. The difference is illustrated in the specific example of Korean traditional art. 


\section{The Special Context of Pansori}

Pansori (Korean also transliterated as p'ansori) is a genre of Korean traditional music. It is a vocal and percussional music performed by one sorikkun (Korean a singer) and one gosu (a drummer playing a barrel drum called buk Korean). The term pansori is derived from pan (Korean, meaning "a place where many people gather"), and sori (Korean meaning "sound": from http://en.wikipedia.org/wiki/Pansori, Feb. 8th. 2015).

The most remarkable part about Pansori is the fact that the singer sings for about 5 to 6 hours by $\mathrm{him} / \mathrm{h}$ erself only supported by a drum. To sing a song for such a long time is not easy for anyone, even for someone who is a highly skilled, well-trained professional singer. According to the Pansori singers, there is a special phase in their performances similar to "time distortion" in flow.

The Pansori singers say that the secret to be able to sing for such a long time is the following: After the performance starts, the singers try to get into rhythm with their drummers for about an hour. When their interactions reach the perfect balance, the singers enter into a state of ecstasy. At this very moment, they experience Shinmyeong. According to the singer's explanation, there is no self-awareness. Because there is no "self" who feels tired, they are able to continuously sing as long as they want (Choi, 2000). Even though the state of Shinmyeong seems to be similar to the state of flow, the main condition the singers report is thus not the same. While flow is explained by individual processes, harmony with other people is the most important condition for the state of Shinmyeong. This might be the main difference between flow and Shinmyeong. Of course, some individual processes such as the match of skill and challenge will also influence the experience of the Pansori singers. They are indeed well-trained professional artists. However, the singers explain the moment they get into the state of Shinmyeong as the moment they reach the perfect tune with their drummer.

So far, the importance of interpersonal processes for the Shinmyeong experience, which seems to share many aspects with flow, has been repeatedly elaborated. We will thus have a closer look at these processes and how they relate to the flow experience.

\section{Understanding Interpersonal Processes in Shinmyeong}

Shinmyeong basically is an individual experience. However, it becomes collective through interpersonal processes, that is, interactions among people who are sharing experiences. In summary, the initial feelings of Shinmyeong are based on recognizing one's own value, which is then influenced by the existence of others and interactions with them. This influence makes Shinmyeong a collective experience that grows bigger. People display the increasing feelings on the outside through various expressive activities, which are again influenced by others. This interplay characterizes the experience of Shinmyeong, even in highly individualistic activities. These interpersonal processes (Intervening condition and Action/Interaction, see Figure 1) lead to an escalation of feelings, ending in an extreme state that is very similar to flow.

However, in the Intervening Condition a person who is carrying out a task is sharing feelings with other 
people who are in the same place. Irrespective of personally knowing each other, the main characteristic of this process in the Shinmyeong situation is co-experience. Co-experience makes sharing of feelings possible and lets people have the same experience. This co-experience becomes possible by a subjective feeling. For example, the person who is co-experiencing something with other people might think "I am feeling what they are feeling, or I exactly know what they think, what they want to do and how they do it". When this moment comes, people get into the peak state of Shinmyeong. In the case of artistic performances, the performer who reaches this peak state can engage in creative activities with the perfect psychological support from the audience that caused this process (Han, 2010).

This psychological process can be understood with the help of Shimcheong communication, the Korean cultural way of communicating. Koreans are accustomed and sensitive to accept other people's feelings as their own. They tend to subjectify other's thoughts, feelings, intentions, etc. when they communicate with them. The subjectified psychological experience of others is called "Shimcheong" (Choi \& Kim, 1999). In fact, Shimcheong communication is possible only when the people involved already have something to share, such as memories of family members. However, Korean people tend to extend the range of personal relationships from family to other in-groups. This extended group is named as "Woori" (we). The main method of communication in "we" is Shimcheong. "We" only remains as a gathering of individuals, if they do not exchange Shimcheong (Choi \& Choi, 1994).

People who experience Shinmyeong begin to exchange their feelings in similar ways as observed in Shimcheong communication. The same happens in the processes of the Intervening condition and other interactions. Experiencing Shinmyeong, people try to confirm whether their feelings are appropriate and acceptable. On account of the attribute of co-experience, people who are at the same place and time believe that they are sharing the same feelings. Through checking the reactions from others, they feel free to do anything they want. This process can amplify the amount of feelings of Shinmyeong. This process is "feedback", which is not new because Csikszentmihalyi mentioned it as preconditions for flow with "clear goal" and "the balance of challenge and skill". Csikszentmihalyi (1988) explained that clear goals and continuing feedback during one's activity can help someone concentrate on work and make it easier to get into the state of flow. However, in this case, the meaning of feedback is limited in the individual perception. For example, while a person is performing a certain task, he checks how the work is going with the help of the information he can get. The checking processes are called "feedback". On the other hand, the interpersonal processes in Shinmyeong include more comprehensive contexts. The individual who is experiencing Shinmyeong would be checking his/her actions continually as the same as flow, that could be said the intrapersonal feedback. At the same time, he/she focuses on the reactions from the people who are sharing the same situation and interplay with them, hence the interpersonal feedback. The individual's experience could be influenced by these two kinds of feedback simultaneously. Especially in the context of everyday life, there exist a lot of interpersonal interactions that influence individual's performance quality. Thus, interpersonal processes are crucial in 
flow experience.

\section{Conclusion}

The concept of flow has made great contributions to the studies on happiness. However, the theory of flow has shortcomings especially in regard to the explanation on the interpersonal processes during experiencing flow. Most activities people experience everyday are closely related and constantly intervened by the existence of others. The mere existence of others itself could influence one's behaviors. Moreover, the interactions not only between the person who is participating in an activity and the others who are related with him/her or the activity but also among the people except for the one who is committed an activity could. On top of one's individual performance, all these interactions together construct one's experience. To further understand the concept of flow and to find more various ways to be happy, it is necessary to consider another explanation on getting into the state of flow-besides the one Csikszentmihalyi (1988) suggested. With the case of Shinmyeong, in some sense, as a cultural variation of flow, there is a possible answer, the influence of interpersonal processes. People can experience flow through the interactions with others as well as the activities.

Of course, the reason why the concept of flow has been considered so valuable in respect of human happiness thus far would be that it has been dealing with the way through which an individual can reach his own happiness within his abilities (Csikszentmihalyi, 1991). However, if the ways to reach great happiness could be various, the discussions about the interpersonal processes in flow would be valuable, especially in terms of the values Csikszentmihalyi (2004) mentioned as the characteristics that could help people experience flow in everyday situations such as altruism and empathy. These are expected to be related not to intrapersonal processes of flow but to the interpersonal processes.

Shinmyeong has been known as the most ideal psychological experience in Korean culture. Korean people named Shinmyeong all the positive experiences they could possibly experience even in the individualistic activities, including the ones that have been mentioned in flow studies (Han, 2009). In other words, it is important to note that the concept of Shinmyeong has been developed in the ordinary context and it occurs with interpersonal interaction.

Therefore, the interpersonal processes discussed with the examples of Shinmyeong are applicable to the study of flow. Of course, further examination is required to verify how many cultural experiences similar to flow exist and how people experience them. Because the cultural concepts comparable to flow might have been constructed culturally, it is necessary to understand the meanings of the phenomena and the ways of experiencing them - as sought in this article. With the results of such works, the concept of flow becomes more useful to help people reach the greater happiness. 


\section{References}

Choi, J. S. (2002). Why don't Korean like regulations? Looking for aesthetic of irregularity. Korean, Seoul: Sonamoo.

Choi, S. C., \& Choi, S. H. (1994). We-ness: A Korean discourse of collectivism. In G. Yoon, \& S. C. Choi (Eds.), Psychology of the Korean people (pp. 57-84). Seoul: Dong-A Publishing \& Printing Co., Ltd.

Choi, S. C., \& Kim, K. (1999). The Shimcheong psychology: Psychological characteristics, interactions, and development of Shimcheong. The Korean Journal of Psychology, 18(1), 1-16.

Csikszentmihalyi, M. (1988). Introduction in Optimal Experience Psychological Studies of Flow in Consciousness. UK, Cambridge: Cambridge University.

Csikszentmihalyi, M. (1991). Flow: The psychology of optimal experience. New York: Harper Prennial.

Csikszentmihalyi, M. (1998). Finding flow: The Psychology of Engagement with Everyday Life. Oshkosh, WI: Basic book.

Csikszentmihalyi, M. (2000). Beyond Boredom and Anxiety: Experiencing Flow in Work and play. San Francisco, CA: Jossey-Bass.

Csikszentmihalyi, M. (2004). Good business: Leadership, flow, and the making of meaning. UK, Westminster: Penguin.

Han S. (1988). The relationship between life satisfaction and flow in elderly Korean immigrants. In M. Csikszentmihalyi, \& I. S. Csikszentmihalyi (Eds.), Optimal Experience: Psychological Studies of Flow In Consciousness (pp. 138-149). Cambridge University Press..

Han, M., \& Han, S. Y. (2007a). A Study of conceptualizing Shinmyeong. Korean Journal of Psychology, 26(1), 83-103.

Han, M., \& Han, S. Y. (2007b). A qualitative study of conceptualizing Shinmyeong. Korean Journal of Psychology, 26(2), 279-306.

Han, M. (2009). The psychology of Shinmyeong. Korean, Seoul: 21th century books.

Han, M. (2010). How can creativity in social context be possible? Culture \& Psychology, 16(2), 165-173.

Kim, K. H., Choi, K. Y., Lee, S. L., \& Seo (1982). A comprehensive study on Korean Shamanism. Korean, Seoul: Research Institute of Korean Studies.

Massimini, F., \& Carli, M (1988). The Systematic Assessment of Flow in Daily Experience. In M. Csikszentmihalyi, \& I. S. Csikszentmihalyi (Eds.), Optimal Experience: Psychological Studies of Flow In Consciousness (pp. 266-287). Cambridge University Press.

Oh, Y. J. (1995). A study of Shinmyung experience in Dance. The Korean Journal of Dance, 18, 161-172.

Sato, I. (1988). Bosozoku: Flow in Japanese motorcycle gangs. In M. Csikszentmihalyi, \& I. S. Csikszentmihalyi (Eds.), Optimal Experience: Psychological Studies of Flow In Consciousness 
(pp. 92-117). Cambridge University Press.

Seligman, M. E. P., \& Csikszentmihalyi, M. (2000). Positive Psychology: An Introduction. American Psychologist, 55(1), 5-14.

Strauss, A., \& Corbin, J. (1998). Basic of Qualitative Research. New Deli: Sage publications.

Tamura, M., \& Mugishima, F. (1975). Bosozoku no Jittai Bunseki [The present state of motorcycle gangs]. Kagaku Keisatsu Kenkyujo Hokoku, 16, 38-72.

Yoo, J., \& Kim, J. W. (2004). Shinmyung Sources Experienced by Professional Korean Dancers. Korean Journal of Sports Psychology, 15(1), 97-112.

World-famous Korean. (1994). A separate booklet appended to the Monthly Chosun. [Korean] 